Article

\title{
Equivalent Electrical Circuits of Thermoelectric Generators under Different Operating Conditions ${ }^{\dagger}$
}

\author{
Saima Siouane, Slaviša Jovanović and Philippe Poure *
}

Institut Jean Lamour (UMR7198), Université de Lorraine, BP 70239, F-54506 Vandoeuvre lès Nancy, France; saima.siouane@univ-lorraine.fr (S.S.); slavisa.jovanovi@univ-lorraine.fr (S.J.)

* Correspondence: philippe.poure@univ-lorraine.fr; Tel.: +333-83-68-41-60

$+\quad$ This paper is an extended version of our paper published in Siouane, S., Jovanović, S. and Poure, P. Equivalent electrical circuit of thermoelectric generators under constant heat flow. In Proceedings of the 2016 IEEE 16th International Conference on Environment and Electrical Engineering (EEEIC 2016), Florence, Italy, 7-10 June 2016.

Academic Editor: Rodolfo Araneo

Received: 10 February 2017; Accepted: 15 March 2017; Published: 18 March 2017

\begin{abstract}
Energy harvesting has become a promising and alternative solution to conventional energy generation patterns to overcome the problem of supplying autonomous electrical systems. More particularly, thermal energy harvesting technologies have drawn a major interest in both research and industry. Thermoelectric Generators (TEGs) can be used in two different operating conditions, under constant temperature gradient or constant heat flow. The commonly used TEG electrical model, based on a voltage source in series with an electrical resistance, shows its limitations especially under constant heat flow conditions. Here, the analytical electrical modeling, taking into consideration the internal and contact thermal resistances of a TEG under constant temperature gradient and constant heat flow conditions, is first given. To give further insight into the electrical behavior of a TEG module in different operating conditions, we propose a new and original way of emulating the above analytical expressions with usual electronics components (voltage source, resistors, diode), whose values are determined with the TEG's parameters. Note that such a TEG emulation is particularly suited when designing the electronic circuitry commonly associated to the TEG, to realize both Maximum Power Point Tracking and output voltage regulation. First, the proposed equivalent electrical circuits are validated through simulation with a SPICE environment in static operating conditions using only one value of either temperature gradient or heat flow. Then, they are also analyzed in dynamic operating conditions where both temperature gradient and heat flow are considered as time-varying functions.
\end{abstract}

Keywords: thermoelectric generator; equivalent electrical circuit; electrical modeling; constant temperature gradient; constant heat flow

\section{Introduction}

In the last decade, many research works have been focused on the design of autonomous systems with capability to operate uninterruptedly. For instance, the wireless sensors used in medical applications require autonomous power sources capable of powering nodes with expected lifetimes exceeding 10 years [1,2]. The low power consumption of these systems is desirable but not sufficient to ensure the continuity of their operation during time. Energy harvesting may come as a solution to ensure the energy provision from the environment and that way extend the lifetime of these systems [3]. Energy harvesting is a process allowing to extract small amounts of available energy from an environment (industrial or domestic) that would otherwise be lost, under different forms such as heat, light or vibrations. If these small amounts of harvested energy are processed and used 
advisedly, the power ratings, especially of autonomous embedded systems, would be much lower. Recently, the energy harvesting from heat attracted a considerable attention due to its abundance in the environment. Heat is converted into electricity through a thermoelectric energy harvesting chain composed of a thermoelectric generator (TEG) and an electronic circuitry, whose main role is to extract a maximum of available electric power and to regulate the output voltage. The thermoelectric generator, the main component of the thermoelectric energy harvesting chain, uses Seebeck effect to convert heat directly into electrical energy [4,5]. The microfabrication of thermoelectric generators has already been reported for powering autonomous wireless sensors in and around the human body in [6,7]. In [7], the TEG harvester is used to supply an autonomous wireless sensor, which is a part of a large body area network, for temperature measurement. Moreover, the use of thermoelectric generators to convert the human body heat into electricity has also been reported in [8], where power levels of 5- $0.5 \mathrm{~mW}$ corresponding to ambient temperatures of $15-27^{\circ} \mathrm{C}$, have been obtained respectively.

A thermoelectric generator consists of $\mathrm{N}$ pairs of $\mathrm{p}$ and $\mathrm{n}$ semiconductors, connected electrically in series and thermally in parallel as shown in Figure 1. One of the main characteristics of a TEG is the absence of moving parts, making it very robust and desirable for high reliable energy harvesting systems $[9,10]$. Moreover, the recent research studies show a stimulated interest in developing inexpensive and eco-friendly thermoelectric materials used for TEG microfabrication, making a TEG a good candidate for supplying autonomous wireless sensors [11].

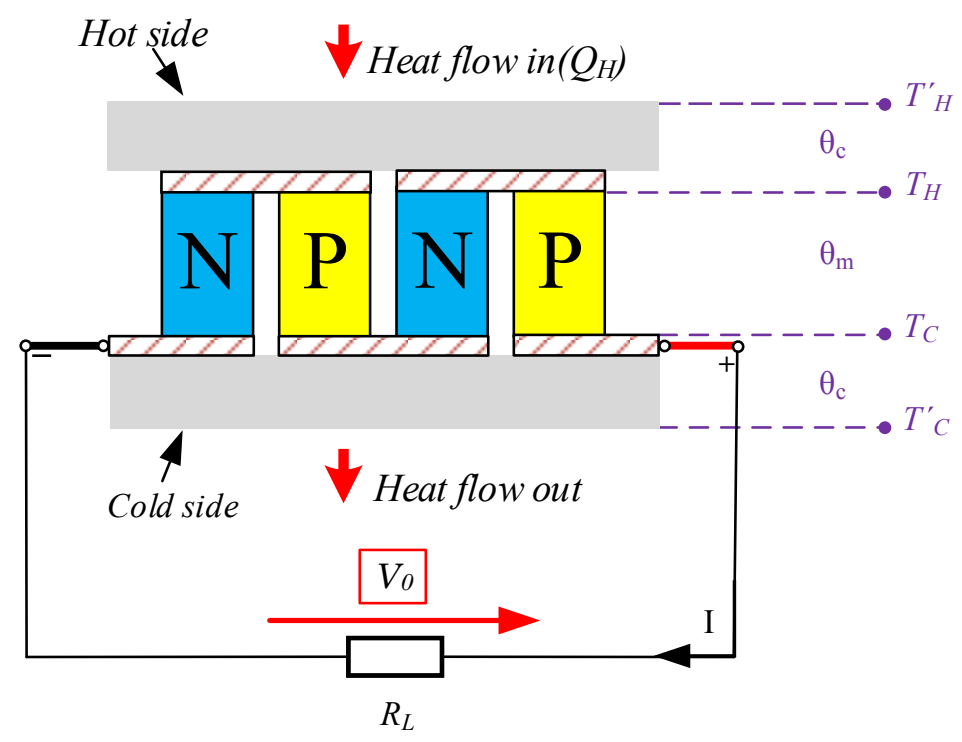

Figure 1. General structure of a Thermoelectric Generator (TEG).

The main parameters defining a TEG are listed bellow:

- The Seebeck coefficient $\alpha=\alpha_{p}-\alpha_{n}$, which depends on the thermoelectric materials used to manufacture p-n semiconductors.

- The electrical resistance $R_{E}$, comprising the electrical resistance of the p-n semiconductors and contacts used to connect the TEG to an electrical load.

- The internal thermal resistance $\theta_{m}$ and the contact thermal resistance $\theta_{c}$, used to interface the TEG to a thermal source of energy.

Different physical models of thermoelectric generators have been reported in the scientific literature [12]. The reported models bring to the foreground electro-thermal coupling, taking into account the above parameters, but are not fully electrical and thus are difficult to use within electrical circuit simulators without considerable design efforts. Indeed, the most used fully electrical model of a TEG in the literature is the commonly used one composed of a constant voltage source $\alpha \Delta T$ in series with a constant electrical resistance $R_{E}$, which neglects the thermal resistances $\theta_{m}$ and $\theta_{c}$. 
Moreover, this same electrical model is used under both constant temperature gradient and constant heat flow operating conditions. Therefore, the fully electrical models of a thermoelectric generator under different operating conditions which take into consideration all above mentioned parameters are needed. This paper is organized as follows: in Section 2, the analytical electrical modeling, taking into consideration the thermal resistances of a TEG under constant temperature gradient and constant heat flow conditions, is given; then, Section 3 presents a new way of emulating the analytical electrical models with equivalent electrical circuits describing faithfully the behaviour of a TEG for both conditions, facilitating that way the design of electronic circuit interfaces needed for real applications. The proposed equivalent electrical circuits are validated through simulation with a SPICE environment whose results are also presented in this section for static operating conditions using one value of either temperature gradient $\Delta T^{\prime}$ or heat flow $Q_{H}$. In Section 4, the proposed equivalent electrical circuits are also analyzed in dynamic operating conditions where both temperature gradient $\Delta T^{\prime}$ and heat flow $Q_{H}$ are considered as time-varying functions. Finally, some conclusions and future work are discussed in Section 5.

\section{Fully Electrical Modeling of a TEG under Different Operating Conditions}

\subsection{Constant Temperature Gradient Conditions}

In the literature, TEGs are mostly considered under constant temperature gradient conditions $[13,14]$. The influence of the internal contact thermal resistance $\theta_{c}$ is usually neglected. Under these conditions, a TEG can be analytically modeled with an equivalent constant voltage source in series with an equivalent internal resistance $[15,16]$ given by:

$$
\begin{gathered}
\left.V_{e q}\right|_{\Delta T^{\prime}=c n s t, \theta_{c}=0}=\alpha \Delta T^{\prime}=\alpha \Delta T \\
\left.R_{e q}\right|_{\Delta T^{\prime}=c n s t, \theta_{c}=0}=R_{E}
\end{gathered}
$$

with $\Delta T^{\prime}=T_{H}^{\prime}-T_{C}^{\prime}, \Delta T=T_{H}-T_{C}$, where $T_{H}^{\prime}, T_{C}^{\prime}$ are respectively the external temperatures of the hot and cold sides of a TEG module, $T_{H}$ and $T_{C}$ are the hot and cold temperatures "seen" by the TEG (see Figure 1). However, in a more accurate model, the real internal contact thermal resistance $\theta_{c}$ between the $\mathrm{p}$ and $\mathrm{n}$ semiconductor elements and their metal contacts must be taken into account. In recent studies, the influence of the internal contact resistance $\theta_{c}$ on the TEG electrical model is demonstrated [17,18], where a slightly similar model to the commonly used one (Equations (1) and (2)) is provided. With $\theta_{c} \neq 0$, the TEG electrical model's parameters depend also on the value of this internal contact resistance and the TEG's thermal parameters. Accordingly, the Equations (1) and (2) become [17]:

$$
\begin{gathered}
\left.V_{e q}\right|_{\Delta T^{\prime}=c n s t, \theta_{c} \neq 0}=\alpha \Delta T^{\prime} \frac{\theta_{m}}{\theta_{m}+2 \theta_{c}} \\
\left.R_{e q}\right|_{\Delta T^{\prime}=c n s t, \theta_{c} \neq 0} \approx R_{E}+\frac{\alpha^{2} \theta_{c} \theta_{m}\left(T_{H}^{\prime}+T_{C}^{\prime}\right)}{\theta_{m}+2 \theta_{c}}
\end{gathered}
$$

Equations (3) and (4) show that the TEG electrical model's parameters $V_{e q}$ and $R_{e q}$ are not only dependent on the TEG's thermal parameters $\alpha, \theta_{c}, \theta_{m}$ and $R_{E}$, but also evolve with the temperature gradient applied to the TEG's terminals. This is especially the case of $R_{e q}$, which for given TEG's thermal parameters can no longer be considered as constant, due to the variation of $T_{H}^{\prime}$ with the temperature gradient $\Delta T^{\prime}$. 


\subsection{Constant Heat Flow Conditions}

In some practical applications, as in the case of automobile exhaust gas thermal energy recovery systems [19], TEGs are subject to a constant thermal input heat flow instead of a constant temperature gradient. Under constant heat flow conditions, with the contact thermal resistance neglected, the TEG can be electrically modeled with an equivalent constant voltage whose analytical expression is given by Equation (5), in series with an equivalent internal electrical resistance whose analytical expression is given by Equation (6) [18]:

$$
\begin{gathered}
\left.V_{e q}\right|_{Q_{H}=c n s t, \theta_{c}=0}=\alpha \theta_{m} Q_{H} \\
\left.R_{e q}\right|_{Q_{H}=\text { cnst } \theta_{c}=0} \approx R_{E}+\alpha^{2} Q_{H} \theta_{m}^{2}+\alpha^{2} \theta_{m} T_{C}^{\prime}-\left[\frac{\alpha R_{E} \theta_{m}}{2}+\alpha^{3} \theta_{m}^{2} T_{C}^{\prime}+\alpha^{3} \theta_{m}^{3} Q_{H}\right] I \\
+\left[\alpha^{4} \theta_{m}^{3} T_{C}^{\prime}+\alpha^{4} Q_{H} \theta_{m}^{4}+\frac{\alpha^{2} R_{E} \theta_{m}^{2}}{2}\right] I^{2}
\end{gathered}
$$

Equations (5) and (6) show that the internal equivalent resistance of the TEG $R_{\text {eq }}$, even in the case $\theta_{c}=0$, depends on the TEG's parameters $\alpha, \theta_{m}$ and $R_{E}$, and on the load current $I$. This was not the case for the TEG with $\theta_{c}=0$ under constant temperature gradient conditions (Equation (2)) whose model is commonly used in electrical simulations. Moreover, the current-dependent terms of $R_{e q}$ under constant heat flow conditions presented in Equation (6) cannot be neglected due to the higher orders of current dependency. On the other hand, when the contact thermal resistance $\theta_{c}$ is considered, the TEG under constant heat flow conditions can be modeled with the same equivalent constant voltage source in series with an internal electrical resistance whose expressions are given by [18]:

$$
\begin{gathered}
\left.V_{e q}\right|_{Q_{H}=c n s t, \theta_{c} \neq 0}=\alpha \theta_{m} Q_{H} \\
\left.R_{e q}\right|_{Q_{H}=c n s t, \theta_{c} \neq 0} \approx R_{E}+\alpha^{2} \theta_{c} \theta_{m} Q_{H}+\alpha^{2} \theta_{m} T_{C}^{\prime}+\alpha^{2} \theta_{m}^{2} Q_{H}-\left[\frac{\alpha R_{E} \theta_{m}}{2}+\alpha^{3} \theta_{m}^{2} T_{C}^{\prime}+\alpha^{3} \theta_{m}^{3} Q_{H}\right. \\
\left.+2 \alpha^{3} \theta_{m}^{2} \theta_{c} Q_{H}\right] I+\left[\alpha^{4} \theta_{m}^{3} T_{C}^{\prime}+\alpha^{4} Q_{H} \theta_{m}^{4}+\frac{\alpha^{2} R_{E} \theta_{m}^{2}}{2}+\alpha^{2} R_{E} \theta_{m} \theta_{c}+\alpha^{4} \theta_{m}^{2} \theta_{c} Q_{H}\left(\theta_{c}+3 \theta_{m}\right)\right. \\
\left.+\alpha^{4} \theta_{m}^{3} \theta_{c}^{2} T_{C}^{\prime}\right] I^{2}
\end{gathered}
$$

The expression of $R_{e q}$ given in Equation (8) shows that the contact thermal resistance $\theta_{c}$ influences both the current-independent (the first 4 terms of Equation (8)) and current-dependent terms. It does mean that even in the absence of load current (open circuit conditions), the equivalent resistance of the TEG module under constant heat flow conditions in the case of $\theta_{c} \neq 0$ (Equation (8)) is higher than in the case of $\theta_{c}=0$ (Equation (6)). Notice that this is not the case under constant temperature gradient conditions, where the equivalent resistance in open circuit case is the same whatever the $\theta_{c}$ value (Equations (1) and (2)).

\section{Equivalent Electrical Circuits of a TEG: Static Operating Conditions with Constant $\Delta T / Q_{h}$}

\subsection{Constant Temperature Gradient Conditions}

The analytical expressions of the equivalent electrical voltage sources $V_{e q}$ and internal resistances $R_{e q}$ of the TEG for both constant temperature and constant heat flow conditions presented with Equations (1)-(8), with and without taking into consideration the contact thermal resistance $\theta_{c}$, describe faithfully the electrical behaviour of the TEG module under different operating conditions. To give further insight into the electrical behaviour of a TEG module in different operating conditions, we propose to emulate the above analytical expressions with lumped-element circuits (voltage sources, resistors, diodes) whose values are determined with the TEG's parameters. 
The equivalent electrical model of a TEG under constant temperature gradient conditions, where $V_{e q}$ and $R_{e q}$ are given with Equations (1) and (2), and with Equations (3) and (4) for a TEG without and with contact thermal resistance respectively, can be emulated with the circuit presented in Figure 2 . This circuit is directly derived from the analytical expressions (1)-(4). For given TEG's parameters $\alpha$, $\theta_{m}, \theta_{c}$ and $R_{E}$, and temperature gradient $\Delta T$, the voltage source $V_{e q}$ and resistance $R_{e q}$ are constant and load current independent. The proposed electrical circuit can directly be employed in electronic circuit simulators to emulate a TEG module under constant temperature gradient conditions and validate the associated electronic circuit designs.

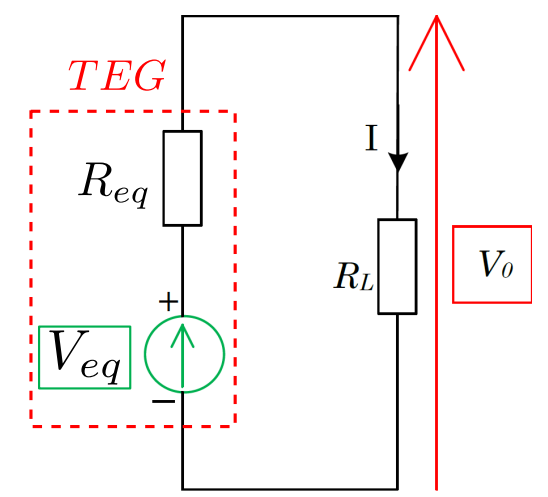

Figure 2. Fully electrical model of a TEG under constant temperature gradient conditions.

\subsection{Constant Heat Flow Conditions}

Similarly, the equivalent electrical model of a TEG under constant heat flow conditions can be emulated with the circuit presented in Figure 3, where $V_{e q}$ and $R_{e q}$ are given with Equations (5) and (6), and with Equations (7) and (8) for a TEG without and with contact thermal resistance respectively. The presented circuit is directly derived from analytical expressions (5)-(8). Unlike the electrical model of a TEG under constant temperature gradient conditions, for given TEG's parameters $\alpha, \theta_{m}, \theta_{\mathcal{c}}$ and $R_{E}$, and heat flow $Q_{H}$, the voltage source $V_{e q}$ is constant and load current independent whereas the resistance $R_{e q}$ is not. From Equation (8), it can be shown that $R_{e q}$ varies between $R_{e q_{\max }}$, corresponding to the value of $R_{e q}$ obtained for $I=0$, and $R_{e q_{\text {min }}}$, corresponding to the value of $R_{e q}$ obtained for $I=I_{\max }$ which is the short-current circuit where $R_{L}=0$. These values, $R_{e q_{\min }}$ and $R_{e q_{\max }}$, are given respectively with:

$$
\begin{gathered}
R_{e q_{\text {max }}}=\left.R_{e q}\right|_{Q_{H}=c n s t, \theta_{c} \neq 0, I=0}=R_{E}+\alpha^{2} \theta_{c} \theta_{m} Q_{H}+\alpha^{2} \theta_{m} T_{C}^{\prime}+\alpha^{2} \theta_{m}^{2} Q_{H} \\
R_{e q_{\text {min }}}=\left.R_{e q}\right|_{Q_{H}=c n s t, \theta_{c} \neq 0, I=I_{S C}}=R_{e q_{\text {max }}}+a I_{S C}+b I_{S C}^{2}
\end{gathered}
$$

where $a, b$ and $I_{S C}$ are defined as:

$$
\begin{gathered}
a=-\left(\frac{\alpha R_{E} \theta_{m}}{2}+\alpha^{3} \theta_{m}^{2} T_{C}^{\prime}+\alpha^{3} \theta_{m}^{3} Q_{H}+2 \alpha^{3} \theta_{m}^{2} \theta_{c} Q_{H}\right) \\
b=\alpha^{4} \theta_{m}^{3} T_{C}^{\prime}+\alpha^{4} Q_{H} \theta_{m}^{4}+\frac{\alpha^{2} R_{E} \theta_{m}^{2}}{2}+\alpha^{2} R_{E} \theta_{m} \theta_{c}+\alpha^{4} \theta_{m}^{2} \theta_{c} Q_{H}\left(\theta_{c}+3 \theta_{m}\right)+\alpha^{4} \theta_{m}^{3} \theta_{c}^{2} T_{C}^{\prime} \\
I_{S C}=\frac{\left.V_{e q}\right|_{Q_{h}=c n s t, \theta_{c} \neq 0}}{R_{e q_{\text {min }}}}
\end{gathered}
$$


In Equation (13), by replacing $V_{e q}$ with Equation (7) and $R_{e q_{\min }}$ with Equation (10), the following expression is obtained:

$$
I_{S C}=\frac{\alpha \theta_{m} Q_{H}}{R_{e q_{\max }}+a I_{S C}+b I_{S C}^{2}} \rightarrow b I_{S C}^{3}+a I_{S C}^{2}+R_{e q_{\max }} I_{S C}-\alpha \theta_{m} Q_{H}=0
$$

$I_{S C}$ from Equation (14) is the only real solution of the 3-order polynomial expression. For the sake of clarity, the full expression of $I_{S C}$ as a function of $a, b, R_{e q}$ max and $V_{e q}$ has been omitted from this work.

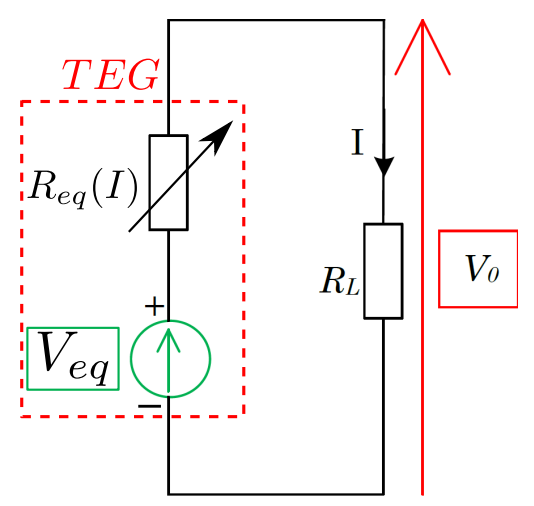

Figure 3. Fully electrical model of a TEG under constant heat flux conditions.

The electrical model of a TEG under constant heat flux conditions presented in Figure 3 is difficult to handle in term of electrical simulation because of the load current dependency. To facilitate the electrical simulation of a TEG under constant heat flow conditions with the thermal contact resistance taken into account, we propose to emulate this load current-dependent resistance. Indeed, the equivalent resistance for any load current under constant heat flow conditions can be presented as:

$$
R_{e q}=\left.R_{e q}\right|_{Q_{H}=c n s t, \theta_{c} \neq 0}=R_{e q_{\max }}+a I+b I^{2}, 0 \leq I \leq I_{S C}
$$

If Equation (15) is closely observed, the current-dependent terms can be considered as a 2nd order MacLaurin series of an exponential function as:

$$
e^{\frac{c_{1}}{c_{2} c_{3}} I}-1 \approx \frac{c_{1}}{c_{2} c_{3}} I+\frac{c_{1}^{2}}{2 \cdot c_{2}^{2} c_{3}^{2}} I^{2}=a I+b I^{2}
$$

where $c_{1}, c_{2}$ and $c_{3}$ are some constant coefficients to determine. Moreover, the non-linear current dependency presented with Equation (16) can be approximatively emulated by the behaviour of a Shockley ideal diode, which is a commonly used in circuit simulators. Notice that this diode is used for electrical simulation purpose only and does not exist physically in the TEG. Therefore, for a TEG operating under constant heat flow conditions, we propose a new and original equivalent electrical circuit, composed of resistances and a non-ideal diode, whose main goal is to emulate the equivalent resistance $\left.R_{e q}\right|_{Q_{H}=c n s t, \theta_{c} \neq 0}$ of Equation (8) [21].

Figure 4 presents this equivalent electrical circuit. The used diode model is a non-ideal model presented in [22] (in red in Figure 4): the resistance $R_{2}$ is a parasitic parallel resistance representing shunt losses at the periphery of the diode, $R_{S}$ is a parasitic series resistance and $D$ is the Shockley ideal diode. Therefore, the diode equation $I_{D}=f\left(V_{D}\right)$ can be represented as follows:

$$
I_{D}=I_{S S}\left(e^{\frac{V_{D}\left(1+\frac{R_{S}}{R_{2}}\right)-I R_{S}}{n V_{t}}}-1\right)
$$


where $n$ is the junction ideality factor, $I_{S S}$ is the junction reverse current, $V_{t}$ is the thermal voltage $k T / q$ where $k$ is the Boltzman's constant, $T$ is the ambient temperature and $q$ is the electron charge. In this study, this thermal voltage is considered constant and equal to $26 \mathrm{mV}$.

The resistance $R_{1}$ and the parameters of the diode $D\left(R_{2}, R_{S}, n, I_{S S}\right)$ are functions of the TEG's physical parameters $\theta_{m}$ and $\theta_{c}$ as well as of the TEG's internal electrical resistance $R_{E}$. They can be identified using Equations (8)-(13) and the expression of the electrical power delivered to the load [18]:

$$
\left.P\right|_{Q_{H}=c n s t, \theta_{c} \neq 0}=V_{O} I
$$

where $V_{O}$ is the voltage at the load's terminals:

$$
V_{O}=V_{e q}-R_{e q} I
$$

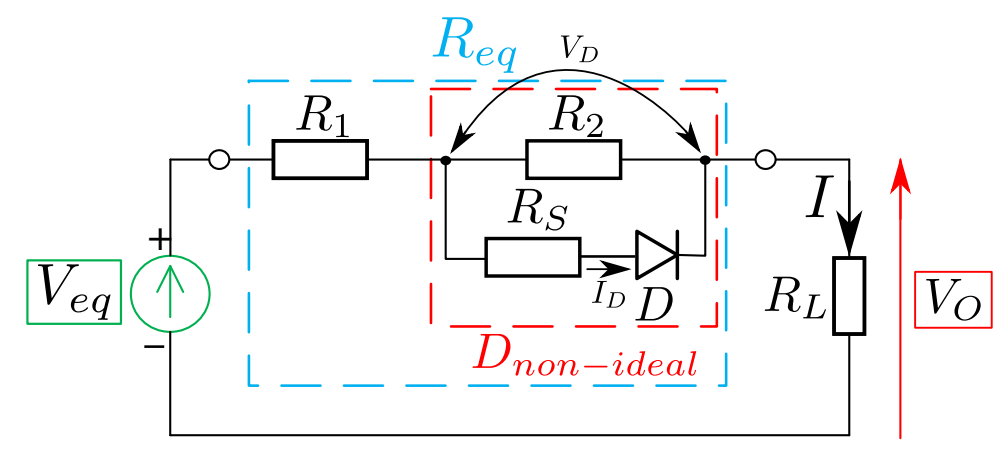

Figure 4. Equivalent electrical circuit of $R_{e q}$ under constant heat flow condition.

The expression of the voltage source $V_{e q}$ used in the proposed equivalent electrical circuit has already been identified in the previous section and presented with Equation (7). This expression is rewritten again and is valid no matter the value of thermal contact thermal resistance $\theta_{c}$ :

$$
V_{e q}=\alpha Q_{H} \theta_{m}
$$

If the equivalent electrical circuit from Figure 4 is analyzed in the case $I=0$, the diode $D$ is not conducting and the equivalent resistance of the circuit is equal to the sum of $R_{1}$ and $R_{2}$ :

$$
R_{1}+R_{2}=\left.R_{e q}\right|_{Q_{H}=c n s t, \theta_{c} \neq 0, I=0}
$$

The resistance given by Equation (21) is equal to the maximal equivalent resistance $R_{e q_{\max }}$ given by Equation (9), thus giving the first relationship between the resistances $R_{1}$ and $R_{2}$ and the TEG's physical parameters:

$$
R_{1}+R_{2}=R_{e q_{\max }}=R_{E}+\alpha^{2} \theta_{c} \theta_{m} Q_{H}+\alpha^{2} \theta_{m} T_{C}^{\prime}+\alpha^{2} \theta_{m}^{2} Q_{H}
$$

The first two parameters of the proposed equivalent electrical circuit are the resistances $R_{1}$ and $R_{2}$. As presented by Equation (22), the sum of these two resistances is equal to $R_{e q_{\max }}$, which is dependent on the TEG's parameters $R_{E}, \alpha, \theta_{c}, \theta_{m}$, the applied heat flow $Q_{H}$ and the temperature of the TEG module's cold side $T_{c}^{\prime}$. To determine these two resistances, some arbitrary values should be assumed at the very beginning. If the diode's voltage value $V_{D}$ is arbitrarily fixed to $0.6 \mathrm{~V}$ when the TEG is short-circuited, then the resistance $R_{1}$ can be calculated as follows:

$$
R_{1}=\frac{V_{e q}-0.6}{I_{s c}}
$$


where $V_{e q}$ and $I_{S C}$ are open-circuit voltage and short-circuit current of a TEG shown in Equations (7) and (13) respectively. It should be stated that other values for diode's voltage at $I_{S C}$ could be used. The value of $0.6 \mathrm{~V}$ is chosen for its similarity to the diode's forward bias voltage. When the $R_{1}$ value is known, the $R_{2}$ value can easily be calculated from Equation (22) as :

$$
R_{2}=R_{e q_{\max }}-R_{1}
$$

In the equivalent electrical circuit presented in Figure 4, the diode parameters should also be determined. The relationship between the current $I$ flowing through the load $R_{L}$ and the diode terminal voltage $V_{D}$ is given by (see Figure 4 ):

$$
I=I_{D}+\frac{V_{D}}{R_{2}}=I_{S S}\left(e^{\frac{\left(\frac{R_{s}}{R_{2}}+1\right) V_{D}-R_{S} I}{n V_{t}}}-1\right)+\frac{V_{D}}{R_{2}}
$$

From Equation (25), the relationship between $n, R_{S}$ and $I_{S S}$ can be established as:

$$
n=\frac{\left(R_{2}+R_{S}\right) V_{D}-R_{S} I R_{2}}{V_{t} R_{2} \log \left(1+\frac{I}{I_{S S}}-\frac{V_{D}}{I_{S S} R_{2}}\right)}
$$

From Equation (26), it can be seen that to establish a relationship between $n, R_{S}$ and $I_{S S}$, the load current $I$ and diode terminal voltage $V_{D}$ should be eliminated. If we replace this couple $\left(I, V_{D}\right)$ by known values, which have to be carefully chosen, the demanded relationship will be established. By putting known values $\left(I_{1}, V_{1}\right)$ and $\left(I_{2}, V_{2}\right)$ in Equation (26), we obtain two equations linking $n$ to $R_{S}$ and $I_{S S}$ :

$$
\begin{aligned}
& n=\frac{\left(R_{2}+R_{S}\right) V_{1}-R_{S} I_{1} R_{2}}{V_{t} R_{2} \log \left(1+\frac{I_{1}}{I_{S S}}-\frac{V_{1}}{I_{S S} R_{2}}\right)} \\
& n=\frac{\left(R_{2}+R_{S}\right) V_{2}-R_{S} I_{2} R_{2}}{V_{t} R_{2} \log \left(1+\frac{I_{2}}{I_{S S}}-\frac{V_{2}}{I_{S S} R_{2}}\right)}
\end{aligned}
$$

from which the relationship between $R_{S}$ and $I_{S S}$, and $n$ and $I_{S S}$ can be established as presented with Equations (29) and (30) respectively.

$$
\begin{aligned}
& R_{S}=-\frac{R_{2} V_{1} \log \left(1+\frac{I_{2}}{I_{S S}}-\frac{V_{2}}{I_{S S} R_{2}}\right)-R_{2} \log \left(1+\frac{I_{1}}{I_{S S}}-\frac{V_{1}}{I_{S S} R_{2}}\right) V_{2}}{\left(V_{1}-I_{1} R_{2}\right) \log \left(1+\frac{I_{2}}{I_{S S}}-\frac{V_{2}}{I_{S S} R_{2}}\right)-\log \left(1+\frac{I_{1}}{I_{S S}}-\frac{V_{1}}{I_{S S} R_{2}}\right) V_{2}+I_{2} R_{2} \log \left(1+\frac{I_{1}}{I_{S S}}-\frac{V_{1}}{I_{S S} R_{2}}\right)} \\
& n=\frac{\frac{I_{1} R_{2}\left(R_{2} V_{1} \log \left(1+\frac{I_{2}}{I_{S S}}-\frac{V_{2}}{I_{S S} R_{2}}\right)-R_{2} \log \left(1+\frac{I_{1}}{I_{S S}}-\frac{V_{1}}{I_{S S} R_{2}}\right) V_{2}\right)}{\left(V_{1}-I_{1} R_{2}\right) \log \left(1+\frac{I_{2}}{I_{S S}}-\frac{V_{2}}{I_{S S} R_{2}}\right)-\log \left(1+\frac{I_{1}}{I_{S S}}-\frac{V_{1}}{I_{S S} R_{2}}\right) V_{2}+I_{2} R_{2} \log \left(1+\frac{I_{1}}{I_{S S}}-\frac{V_{1}}{I_{S S} R_{2}}\right)}}{V_{t} R_{2} \log \left(1+\frac{I_{1}}{I_{S S}}-\frac{V_{1}}{I_{S S} R_{2}}\right)}+ \\
& +\frac{V_{1}\left(R_{2}-\frac{R_{2} V_{1} \log \left(1+\frac{I_{2}}{I_{S S}}-\frac{V_{2}}{I_{S S} R_{2}}\right)-R_{2} \log \left(1+\frac{I_{1}}{I_{S S}}-\frac{V_{1}}{I_{S S} R_{2}}\right) V_{2}}{\left(V_{1}-I_{1} R_{2}\right) \log \left(1+\frac{I_{2}}{I_{S S}}-\frac{V_{2}}{S_{S S} R_{2}}\right)-\log \left(1+\frac{I_{1}}{I_{S S}}-\frac{V_{1}}{I_{S S} R_{2}}\right) V_{2}+I_{2} R_{2} \log \left(1+\frac{I_{1}}{I_{S S}}-\frac{V_{1}}{I_{S S} R_{2}}\right)}\right)}{V_{t} R_{2} \log \left(1+\frac{I_{1}}{I_{S S}}-\frac{V_{1}}{I_{S S} R_{2}}\right)}
\end{aligned}
$$

The biggest issue is to choose the suitable values $\left(I_{1}, V_{1}\right)$ and $\left(I_{2}, V_{2}\right)$ to apply to Equations (27) and (28). To establish a quite accurate model of the TEG under constant heat flow conditions and find its correspondence to the equivalent electrical circuit presented in Figure 4, we want that the I-V curve of the circuit from Figure 4 goes through the Maximum Power Point MPP and finishes at the $I_{S C}$ point. Thus, the couple $\left(I_{1}, V_{1}\right)$ would be the $\left(I_{S C}, 0.6 \mathrm{~V}\right)$ point and the couple $\left(I_{2}, V_{2}\right)$ should 
correspond to the Maximum Power Point $\left(I_{M P P}, V_{M P P}\right)$. The maximum power is obtained by deriving the Equation (18) with regards to I and by finding its real zeros. There are two complex-valued zeros and one real-valued zero which is the $I_{M P P}$ point. The $V_{M P P}$ value is obtained by calculating the voltage at the $I_{M P P}$ value using Equation (19). When the values $V_{M P P}$ and $I_{M P P}$ are known, the second point $\left(I_{2}, V_{2}\right)$ is calculated as follows:

$$
\begin{array}{r}
V_{2}=V_{e q}-R_{1} * I_{M P P}-V_{M P P} \\
I_{2}=I_{M P P}
\end{array}
$$

With these values $\left(I_{1}, V_{1}\right)$ and $\left(I_{2}, V_{2}\right)$, and Equations (29) and (30), by fixing arbitrarily the value of $I_{S S}$, all above mentioned equivalent circuit' parameters can be found.

\subsection{Validation of Equivalent Electrical Circuits through Simulation}

The proposed electrical circuits emulating the TEG under constant temperature gradient conditions (presented in Figure 2) and under constant heat flow conditions (presented in Figure 4) with the parameters given in Tables 1 and 2 are simulated in a SPICE environment and compared to the analytical expressions presented in the previous section in term of power versus the load current $I$.

Table 1. Numerical parameters of the TEG used in simulation

\begin{tabular}{cc}
\hline Parameter & Value \\
\hline $\mathrm{N}$ & 127 \\
$\alpha$ & $0.0531876(\mathrm{~V} / \mathrm{K})$ \\
$R_{E}$ & $1.6(\Omega)$ \\
$\theta_{m}$ & $1.498(\mathrm{~K} / \mathrm{W})$ \\
$\theta_{c}$ & $0.45(\mathrm{~K} / \mathrm{W})$ \\
$T_{C}^{\prime}$ & $298(\mathrm{~K})$ \\
$T_{H}^{\prime}$ & $368(\mathrm{~K})$ \\
$Q_{H}$ & $70(\mathrm{~W})$ \\
\hline
\end{tabular}

Table 2. Numerical parameters and values of the electronic components of the equivalent circuit emulating the TEG (Table 1).

\begin{tabular}{ccc}
\hline Parameter & & Value \\
\hline$V_{e q}$ & $(\mathrm{~V})$ & 5.5787409888 \\
\hline$I_{S C}$ & $(\mathrm{~A})$ & 1.793212008160477 \\
\hline$V_{M P P}$ & $(\mathrm{~V})$ & 2.720928218743778 \\
\hline$I_{M P P}$ & $(\mathrm{~A})$ & 0.8749283875525364 \\
\hline$R_{1}$ & $(\Omega)$ & 0.6648702522882615 \\
\hline$R_{2}$ & $(\Omega)$ & 2.776437457558251 \\
\hline$I_{S S}$ & $(\mathrm{~A})$ & $300 \times 10^{-} 9$ \\
\hline$R_{S}$ & $(\Omega)$ & 0.2016800349713824 \\
\hline$n$ & & 1.084768930249264 \\
\hline$I_{S S}$ & $(\mathrm{nA})$ & 300 \\
\hline
\end{tabular}

These results are presented in Figures 5 and 6. Figure 5 shows that the electrical power of the TEG module obtained with the proposed equivalent electrical circuit $\left(\Delta T^{\prime}=c n s t\right)$ and the one obtained 
with the exact analytical model are superimposed. On the other hand, from Figure 6 it can be seen that the power obtained with the proposed equivalent electrical circuit $\left(Q_{H}=c n s t\right)$ matches very closely the exact one described with the Equation (18). The observed error is below 1\%.

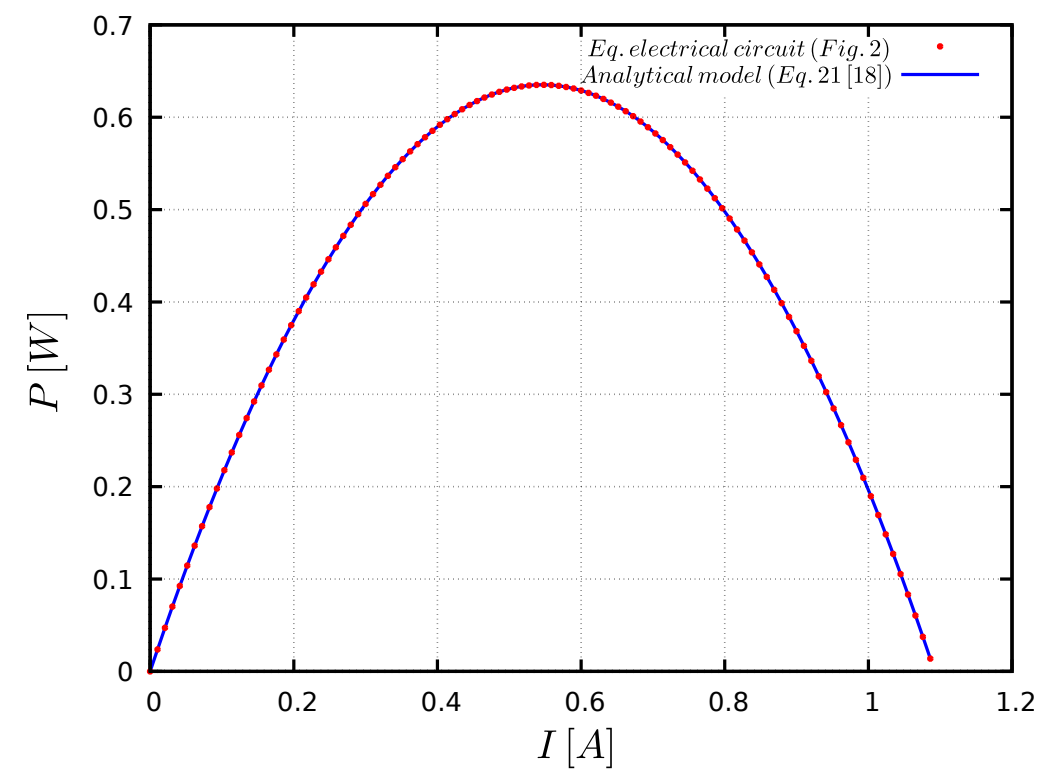

Figure 5. The analytical power and the one obtained from the equivalent electrical circuit of the TEG versus load current under constant temperature gradient conditions $\left(\theta_{c}=0.45 \mathrm{~K} / \mathrm{W}, \Delta T^{\prime}=70 \mathrm{~K}\right)$.

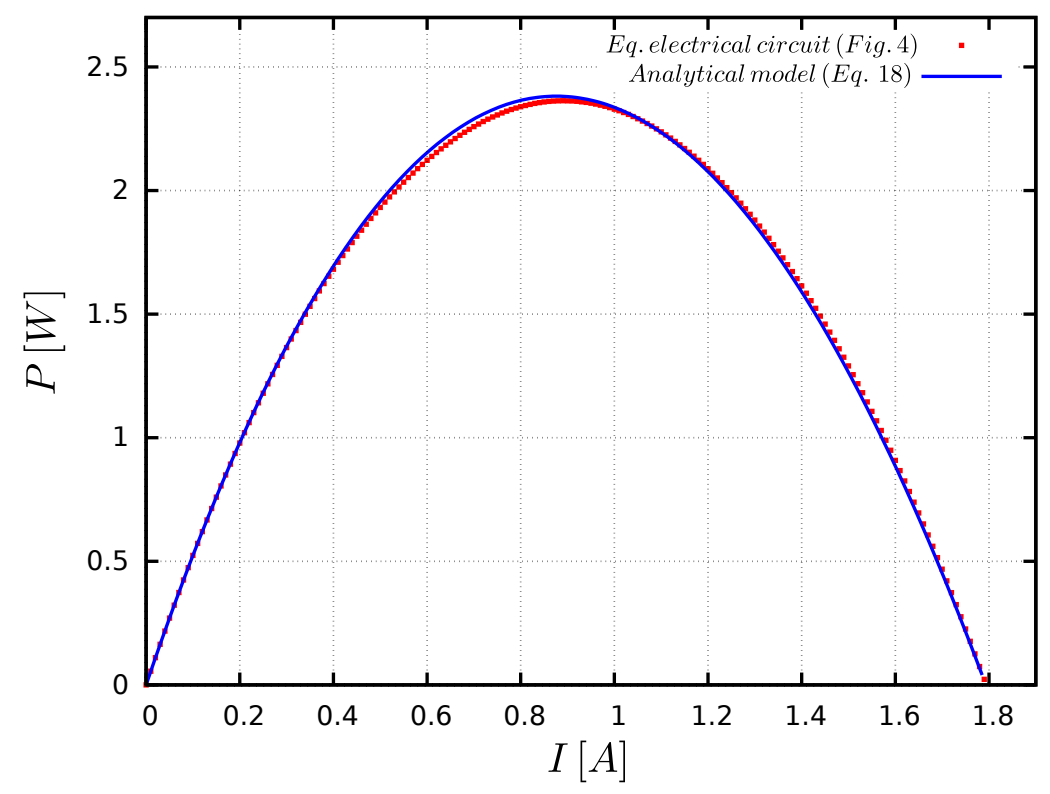

Figure 6. The analytical power and the one obtained from the equivalent electrical circuit of the TEG versus load current under constant heat flow conditions $\left(\theta_{c}=0.45 \mathrm{~K} / \mathrm{W}, Q_{H}=70 \mathrm{~K}\right)$.

The equivalent electrical circuits of a TEG under static operating conditions, where either the temperature gradient between TEG's terminals is kept constant or a constant heat flow applied to it, is summarized in Figure 7 and Table 3. Each operating mode is characterized by its own parameters: the constant temperature gradient by a DC voltage source whose value is directly proportional to $\Delta T$, in series with an equivalent resistance $R_{e q}$ depending on the TEG's parameters and $\Delta T$; the constant heat flow by a DC voltage source whose value is directly proportional to $Q_{H}$, in series with a variable 
resistance block $R_{e q}$ composed of two resistances $R_{1}$ and $R_{2}$ and a diode $\mathrm{D}$ in parallel with the latter, playing the role of the current-dependent resistance.

Table 3. A summary of parameter calculation of the equivalent circuit (Figure 7) under constant $\Delta T^{\prime}$ or $Q_{H}$.

\begin{tabular}{|c|c|c|}
\hline & Parameter & Equation \\
\hline \multirow{2}{*}{$\Delta T^{\prime}=c n s t$} & $V_{e q}$ & $(3)$ \\
\hline & $R_{e q}$ & $(4)$ \\
\hline \multirow{11}{*}{$Q_{H}=c n s t$} & $V_{e q}$ & $(7)$ \\
\hline & $R_{e q_{\max }}$ & $(22)$ \\
\hline & $I_{S C}$ & (14) \\
\hline & $I_{S S}$ & $300 \times 10^{-9}$ \\
\hline & $R_{1}$ & $V_{e q}+I_{S C}+R_{e q_{\max }} \rightarrow(23)$ \\
\hline & $R_{2}$ & $R_{1}+R_{\max } \rightarrow(24)$ \\
\hline & $P_{L}$ & $V_{e q}+(8)+(19) \rightarrow(18)$ \\
\hline & $I_{M P P}$ & $\frac{d P_{L}}{d I}=0$ \\
\hline & $V_{M P P}$ & $I_{M P P} \rightarrow(19)$ \\
\hline & $R_{S}$ & $V_{M P P}+I_{M P P}+I_{S S}+V_{D}+R_{2} \rightarrow(29)$ \\
\hline & $n$ & $V_{M P P}+I_{M P P}+I_{S S}+V_{D}+R_{2}+R_{S} \rightarrow(30)$ \\
\hline
\end{tabular}

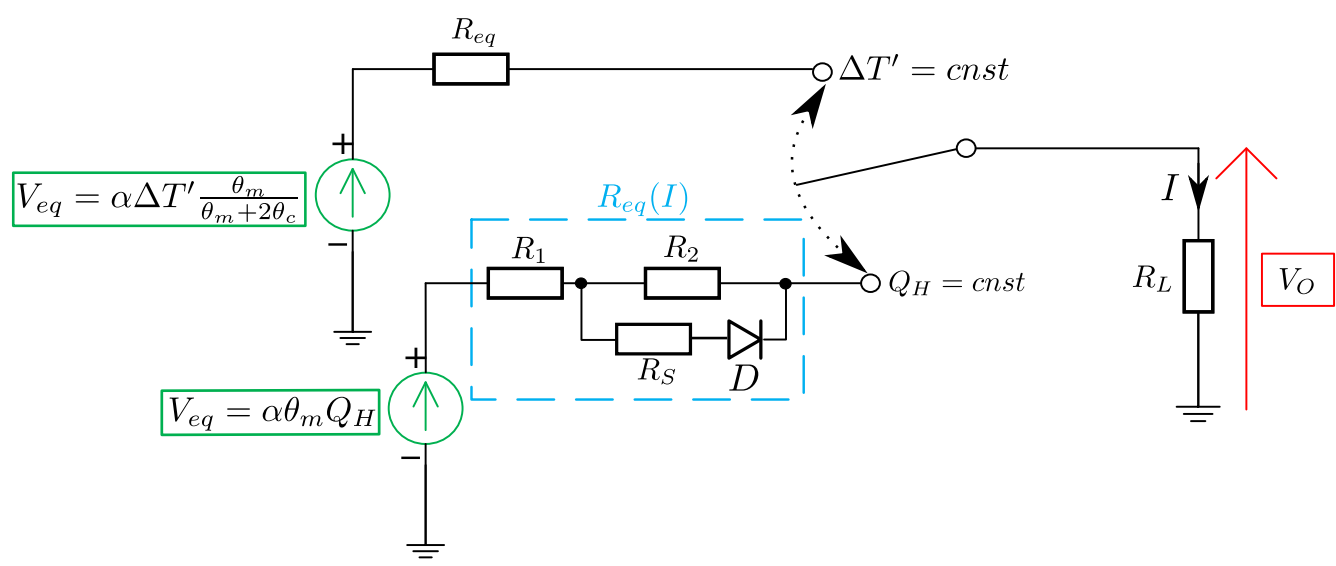

Figure 7. Electrical circuit emulating the TEG under different operating conditions.

\section{Equivalent Electrical Circuits of TEG: Dynamic Operating Conditions $Q_{h}$ or $\Delta T=f(t)$}

The equivalent electrical circuit of the TEG under both constant temperature gradient and constant heat flow conditions studied in the previous section was validated for a given operating point (constant $\Delta T$ in Figure 5 or constant $Q_{H}$ in Figure 6). In this section, the proposed models are evaluated in the dynamic conditions where the values of the temperature gradient $\Delta T^{\prime}$ and heat flow $Q_{H}$ applied to the thermoelectric generator change over time.

In dynamic conditions, the TEG's equivalent parameters $V_{e q}$ and $R_{e q}$ detailed in the previous section for both modes $\left(\Delta T^{\prime}=\right.$ cnst and $Q_{H}=$ cnst $)$ evolve with the values of $\Delta T^{\prime}$ or $Q_{H}$ which are time dependent. Figures 8 and 9 illustrate the behaviour of these models in dynamic conditions for both modes. From these figures, it can be seen that for each operating mode $\left(\Delta T^{\prime}=c n s t\right.$ or $\left.Q_{H}=c n s t\right)$, the parameters $V_{e q}$ and $R_{e q}$ should be recalculated. However, no matter the values of both $\Delta T^{\prime}$ or $Q_{H}$ used in the simulation, the proposed electrical circuits are still valid. 


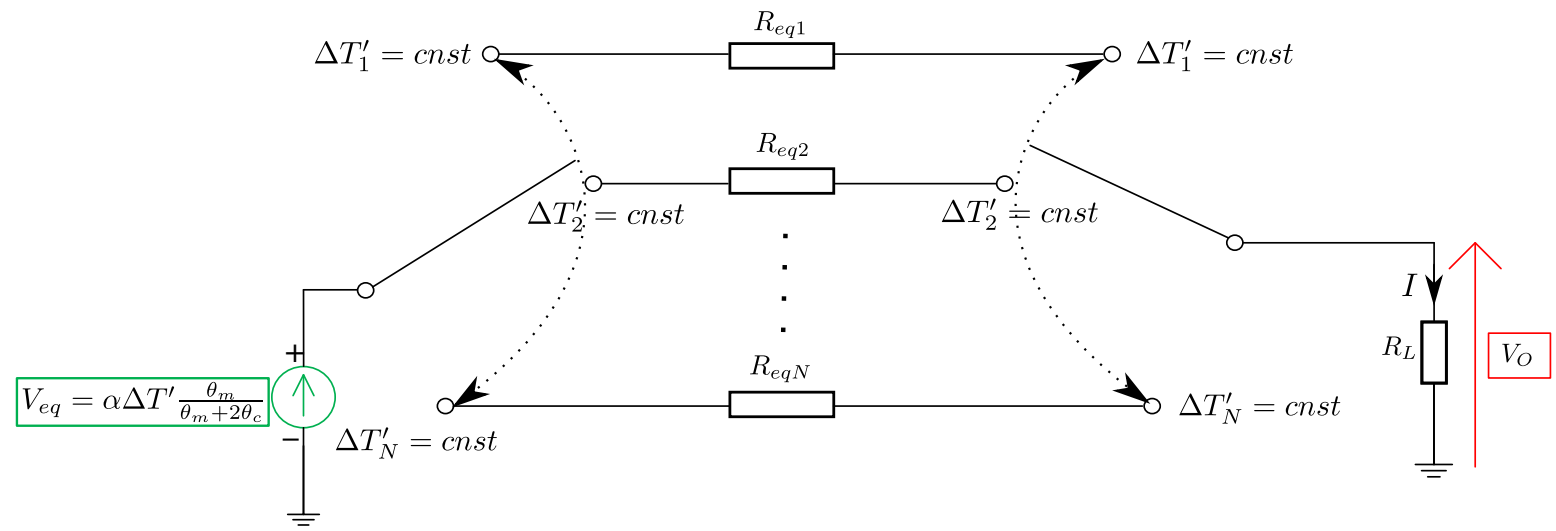

Figure 8. Equivalent electrical circuit of TEG in a dynamic variation under different values of heat flow $Q_{H}$.

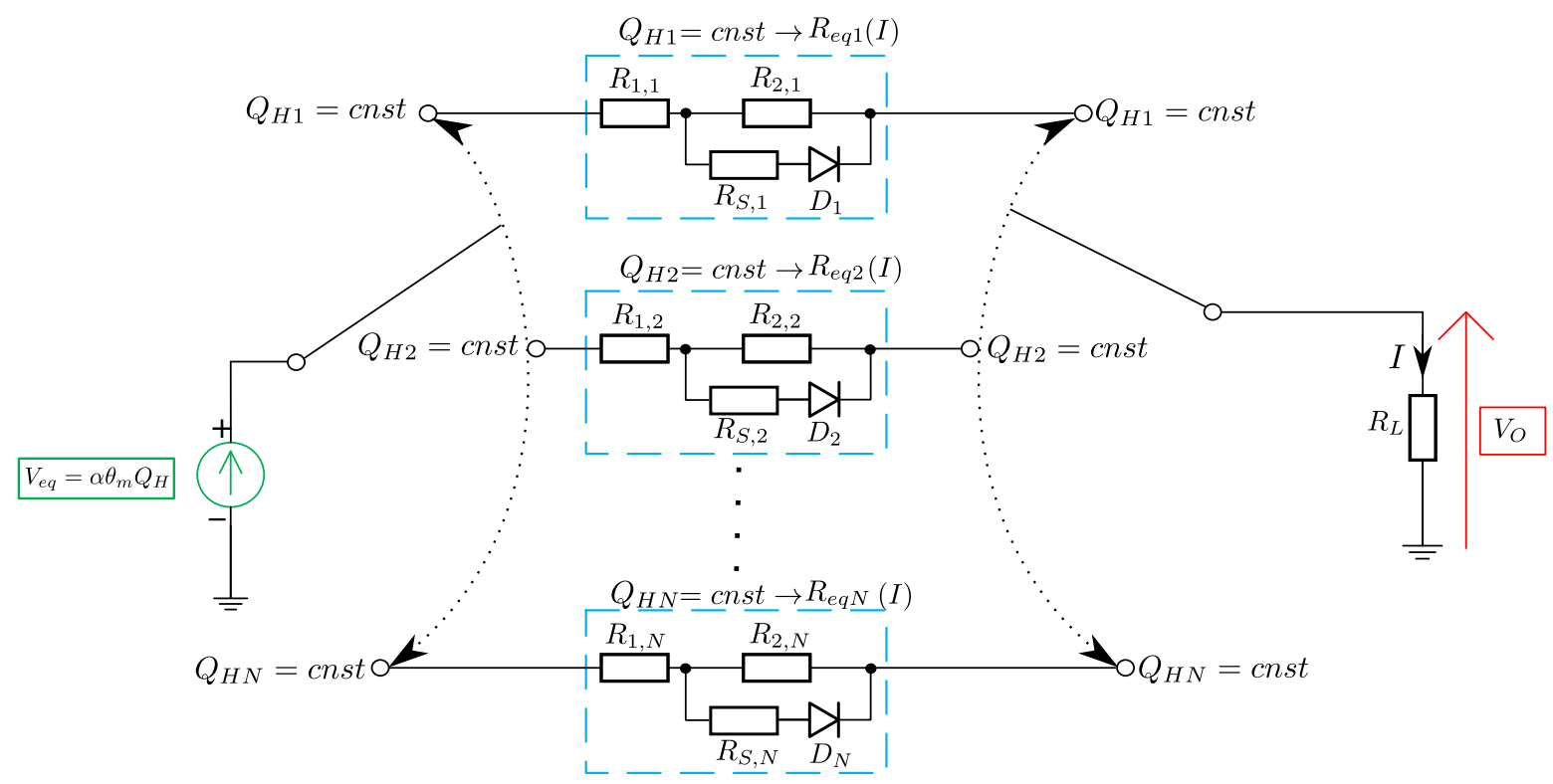

Figure 9. Equivalent electrical circuit of TEG in a dynamic variation under different values of heat flow $Q_{H}$.

Figure 10 shows the evolution of the electrical power delivered to the load by the TEG equivalent circuit in the constant temperature gradient mode under dynamic operating conditions. The electrical power delivered to the load is presented versus load current $I$ for different values of temperature gradient $\Delta T^{\prime}$ (from $10{ }^{\circ} \mathrm{C}$ to $90^{\circ} \mathrm{C}$ ) for a TEG module with the parameters shown in Table 1 . These curves are obtained using the electrical model presented in the previous section, where for each temperature gradient the values of the electrical circuit's parameters, the voltage source $V_{e q}$ and the resistance $R_{e q}$ are calculated again. From Figure 10, it can be seen that the electrical power delivered to the load increases with the temperature gradient increase, which is the expected outcome. Moreover, Figure 11 shows the evolution of this power in the constant heat flow mode also under dynamic operating conditions. The electrical power delivered to the load is also presented versus load current $I$, for different values of heat flow $Q_{H}$ (from $10 \mathrm{~W}$ to $90 \mathrm{~W}$ ) for a TEG with parameters given in Table 1. These curves are similar to those presented in Figure 10. Even in the case of heat flow mode, it can be seen that the output electrical power $P$ delivered to the load increases with the heat flow $Q_{H}$ applied to the TEG. For all curves presented in Figures 10 and 11, the observed error compared with the analytical model is below $1 \%$. 


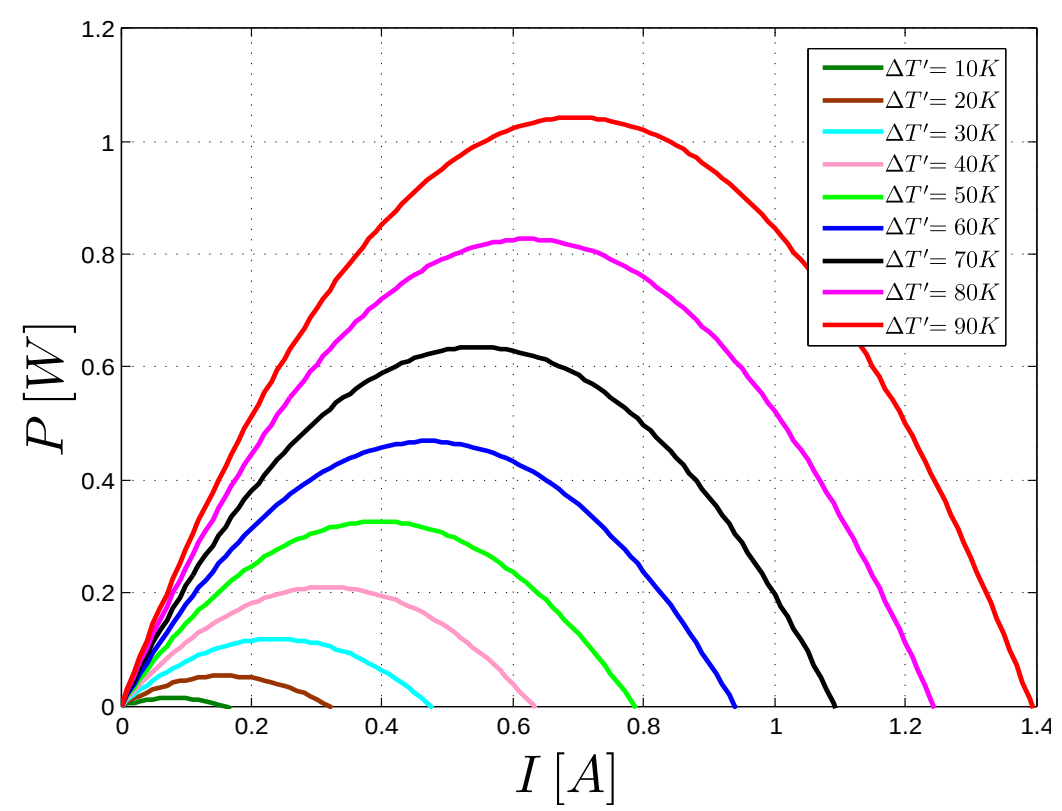

Figure 10. Electrical power as a function of load current $I$ for different values of temperature gradient $\Delta T^{\prime}$.

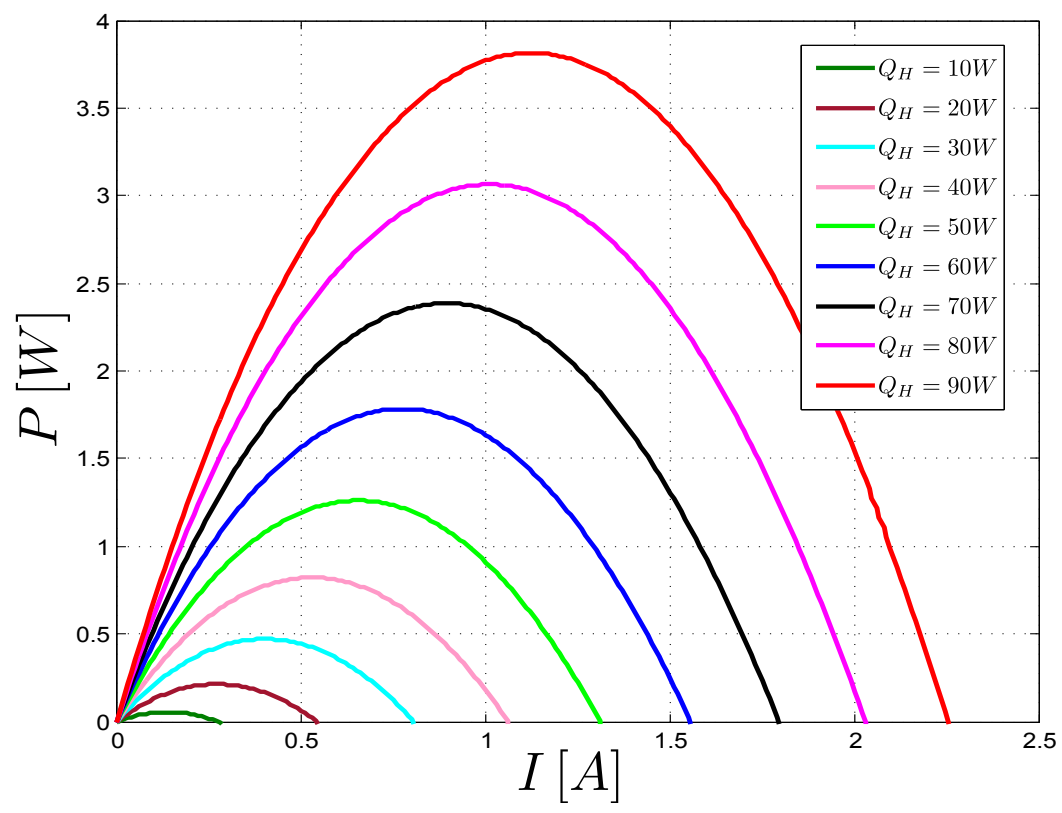

Figure 11. Electrical power as a function of load current $I$ for different values of heat flow $Q_{H}$.

\section{Conclusions}

In this study, the Thévenin equivalent electrical circuits of thermoelectric generators under both constant temperature gradient and constant heat flow conditions were presented. The presented equivalent circuits take into consideration the TEG's internal thermal resistance. Under constant temperature gradient conditions, the voltage source generator proportional to the applied temperature gradient $\Delta T^{\prime}$ on the TEG's terminals in series with an equivalent resistance independent of load current and also linearly dependent on $\Delta T^{\prime}$ is obtained. On the other hand, under constant heat flow conditions, the voltage source generator proportional to the applied heat flow $Q_{H}$ with a load current dependent resistance in series is obtained. Given that the load current dependent resistance of the TEG under heat flow conditions is difficult to handle and simulate, its behaviour was successfully emulated 
using an equivalent electrical circuit comprising a resistance in parallel to a diode. The proposed equivalent electrical circuits were simulated with a SPICE environment and compared to the analytical expressions in terms of power for a given value of either temperature gradient $\Delta T^{\prime}$ or heat flow $Q_{H}$ applied to the TEG. The obtained results show that the power obtained with the proposed electrical circuits matches very closely the exact power expression. The observed error is below $1 \%$. Moreover, the proposed equivalent circuits were tested in dynamic operating conditions where the temperature gradient $\Delta T^{\prime}$ or heat flow $Q_{H}$ across the TEG were considered as time-varying functions. Simulation results show also the validity of the proposed electrical circuits in dynamic operating conditions.

Author Contributions: All authors contributed equally to this work.

Conflicts of Interest: The authors declare no conflict of interest.

\section{References}

1. Mitcheson, P.D.; Yeatman, E.M.; Rao, G.K.; Holmes, A.S.; Green, T.C. Energy harvesting from human and machine motion for wireless electronic devices. Proc. IEEE 2008, 96, 1457-1486.

2. Yang, Y.; Wei, X.-J.; Liu, J. Suitability of a thermoelectric power generator for implantable medical electronic devices. J. Phys. D: Appl. Phys. 2007, 40, 5790.

3. Su, S.; Chen, J. Simulation investigation of high-efficiency solar thermoelectric generators with inhomogeneously doped nanomaterials. Trans. Ind. Electron. 2015, 62, 3569-3575.

4. Roth, R.; Rostek, R.; Cobry, K.; Köhler, C.; Groh, M.; Woias, P. Design and characterization of micro thermoelectric cross-plane generators with electroplated, and reflow soldering. J. Microelectromech. Syst. 2014, 23, 961-971.

5. Dewan, A.; Ay, S.U.; Karim, M.N.; Beyenal, H. Alternative power sources for remote sensors: A review. J. Power Sources Elsevier 2014, 245, 129-143.

6. Koplow, M.; Chen, A.; Steingart, D.; Wright, P.K.; Evans, J.W. Thick film thermoelectric energy harvesting systems for biomedical applications. In Proceedings of the 5th IEEE International Summer School and Symposium on Medical Devices and Biosensors, Hong Kong, China, 1-3 June 2008; pp. 322-325.

7. Kappel, R.; Pachler, W.; Auer, M.; Pribyl, W.; Hofer, G.; Holweg, G. Using thermoelectric energy harvesting to power a self-sustaining temperature sensor in body area networks. In Proceedings of the IEEE International Conference on Industrial Technology (ICIT), Cape Town, South Africa, 25-28 February 2013; pp. 787-792.

8. Leonov, V. Thermoelectric energy harvesting of human body heat for wearable sensors. IEEE Sens. J. 2013, 13, 2284-2291.

9. Ahiska, R.; Mamur, H. Design and implementation of a new portable thermoelectric generator for low geothermal temperatures. Renew. Power Gener. IET 2013, 7, 700-706.

10. Hsu, C.T.; Yao, D.J.; Ye, K.J.; Yu, B. Renewable energy of waste heat recovery system for automobiles. J. Renew. Sustain. Energy 2010, 2, 013105.

11. Carstens, T.; Corradini, M.; Blanchard, J.; Ma, Z. Thermoelectric powered wireless sensors for spent fuel monitoring. In Proceedings of the 2nd IEEE International Conference on Advancements in Nuclear Instrumentation Measurement Methods and their Applications (ANIMMA), Ghent, Belgium, 6-9 June 2011; pp. 1-6.

12. Kim, R.Y.; Lai, J.S.; York, B.; Koran, A. Analysis and design of maximum power point tracking scheme for thermoelectric battery energy storage system. IEEE Trans. Ind. Electron. 2009, 56, 3709-3716.

13. Yazawa, K.; Shakouri, A. Cost-efficiency trade-off and the design of thermoelectric power generators. Environ. Sci. Technol. 2011, 45, 7548-7553.

14. Nemir, D.; Beck, J. On the significance of the thermoelectric figure of merit z. J. Electron. Mater. 2010, 39, 1897-1901.

15. Karami, N.; Moubayed, N. New modeling approach and validation of a thermoelectric generators. In Proceedings of the 2014 IEEE 23rd International Symposium on Industrial Electronics (ISIE), Istanbul, Turkey , 1-4 June 2014; pp. 586-591.

16. Bond, M.; Park, J.D. Current-sensorless power estimation and MPPT implementation for thermoelectric generators. IEEE Trans. Ind. Electron. 2015, 62, 5539-5548. 
17. Siouane, S.; Jovanovic, S.; Poure, P. Influence of contact thermal resistances on the Open Circuit Voltage MPPT method for thermoelectric generators. In Proceedings of the 2016 IEEE International on Energy Conference (ENERGYCON), Leuven, Belgium, 4-8 April 2016.

18. Siouane, S.; Jovanovic, S.; Poure, P. Fully electrical modeling of thermoelectric generators with contact thermal resistance under different operating conditions. J. Electron. Mater. 2017, 46, 40-50.

19. Montecucco, A.; Siviter, J.; Knox, A.R. Constant heat characterisation and geometrical optimisation of thermoelectric generators. Appl. Energy Elsevier 2015, 149, 248-258.

20. Dousti, M.J.; Petraglia, A.; Pedram, M. Accurate electrothermal modeling of thermoelectric generators. In Proceedings of the 2015 Design, Automation \& Test in Europe Conference \& Exhibition (DATE'15), Grenoble, France, 9-13 March 2015; pp. 1603-1606.

21. Siouane, S.; Jovanovic, S.; Poure, P. Equivalent electrical circuit of thermoelectric generators under constant heat flow. In Proceedings of the IEEE 16th International Conference in Environment and Electrical Engineering (EEEIC), Florence, Italy, 7-10 June 2016; pp. 1-6.

22. Ortiz-Conde, A.; Francisco, J.G.; Muci, J. Exact analytical solutions of the forward non-ideal diode equation with series and shunt parasitic resistances. J. Solid-State Electron. 2000, 44, 1861-1864.

(C) 2017 by the authors. Licensee MDPI, Basel, Switzerland. This article is an open access article distributed under the terms and conditions of the Creative Commons Attribution (CC BY) license (http:/ / creativecommons.org/licenses/by/4.0/). 